\title{
Editorial
}

\section{Beauty, Quality and Harmony}

\begin{abstract}
Modeling challenges and modeling possibilities are increasingly involving qualitative rather than simply quantitative pursuits. The world and the worldculture have changed: quality, not just quantity, matters.

Human concerns for preserving ecological, cultural, economic and social resources are undoubtedly going to rise dramatically in the future. Recent examples of widespread destruction, collateral devastation and unwise and misinformed neglect of both natural, built and economic environments should have driven the message home: we have brought most human societies to the very edge of their cultural, economic and even biological sustainability. Yet, new conservation, preservation and enhancement efforts require modes of assessment, judgment and choice for which traditional economic analyses are clearly inadequate.

It would not be a problem to assess things that are singledimensional, i.e., individually the cheapest, the most expensive, the simplest or the easiest to preserve. But we have to preserve things of the entire complex social value [1], things that are beautiful, of the highest quality and of sustainable economic importance. Beauty and quality are not unidimensional concepts like costs, profits or sales.
\end{abstract}

\section{Beauty}

We can all relate to some traditional definitions of beauty, like Aquinas's 'That which pleases the eye (or nose, ear, mouth or touch)', or Adler's 'That which pleases us upon being contemplated'.

Such and similar concepts of beauty are highly subjective: whatever an individual experiences (through whatever senses or processes of contemplation) as beautiful is, at least for him, beautiful. Nothing particularly meaningful can be added here. De gustabus non disputandum est. Such beauty is all 'in the eye of the beholder', a matter of individual perceptual and contemplative taste - extrarational, extrajudgmental and extrascientific.

However, every specific object (system, artifact,

IOS Press

Human Systems Management 11 (1992) 115-118 etc.) is identified as belonging to some gen eric class of objects, to its 'family' or things. Each such object not only belongs to a class, but can also 'occupy a position' within the class. For the purposes of comparison, assessment and judgment we rank, classify, group and order objects within their classes.

Each class of objects can, therefore, be characterized by real or conceived ideal levels of achievement along specific dimensions, criteria, qualities or attributes. From these, standards of excellence, quality or achievement can be derived, forming ideal objects, the most perfect representatives of a given class. These ideals can be either specific and real precedents, or they can be conceived or conceivable composites of specific and real dimensions (or experienced achievements).

Objects which in one way or another conform most perfectly to these ideals are more or less admirable, of high quality, or beautifuls Although individuals may differ in their taste and judgment (or 'measurement') of the conformance of a given specimen to standards of excellence, the classes, the standards and the ideals themselves can be obtained through knowledge, expertise and rational argument: they can be measured and discussed more or less objectively.

The most beautiful rose or flower is generally that which conforms most closely to a particular ideal of a rose within a given class of roses or flow ers. This ideal can sometimes shift or be displaced, but the search for conformance or proximity endures: it serves as a mechanism of human construction (or 'pullback' towards the point of attraction, the ideal) of beauty, quality or admiration.

Beauty is, therefore, related to conspicuous or prominent levels of achievement, like extreme, exaggerated, maximal/minimal, striking, etc. (but also the unusual, the remote, the rare and the 
exotic), at least along some of the dimensions. At the same time, beauty is also related to pattern, symmetry, harmony, completeness, and balance.

The antonym of beauty, ugliness, does not imply 'the other extreme or opposite', but rather mediocrity, plainness, blandness and undistinctiveness, combined with the want of symmetry, with distortion, blemish, deformity or incompleteness.

Ugliness is disordered and asymmetrical arrangement of bland and undistinct achievements. Beauty is harmonious and balanced arrangement of noticeable and distinct achievements. Beauty is negentropic [2].

It is implied that different cultures will differ in their constructions of the ideals, but that beautiful or admirable shall remain across cultures that which most closely resembles or approximates those ideals. It is further proposed that awareness of differential ideals and the universal sense of harmony, symmetry and completeness allows for crosscultural judgments of beauty, at least among the experts and connoisseurs. Many Chinese and Japanese can certainly appreciate the very best Western paintings or Western music, while Americans can exhibit similar appreciation of the very best Chinese screens or Japanese calligraphy.

\section{Multiple Criteria}

Dimensions, attributes, qualities and criteria which may enter into the perception of beauty are not only multiple, but they are also varied and of differential impact and importance. They could also come from separate, even non-intersecting domains.

These dimensions could be primary, secondary or tertiary, they can be quantitative or qualitative, physical, perceptual, artificially constructed or imaginary.

Yet, they should not be aggregated into a single measure.

For example, female beauty is a composite or assemblage of different, unaggregated signs of sexual allurement, characteristics pertaining to bearing, nurturing and caring, like attributes of socialization, stability and reliability, accompanied by the sizes, colors, smells, sounds, textures and adorn- ments that emphasize (or deemphasize) basic definitional traits of a given class of females (universal, racial, type, role, etc.).

It is the organization, harmony and balanced completeness of all these dimensions that produces the perception and the sense of beauty, not their specific and particular structural manifestations (like fairness, darkness, coarseness or smoothness).

Any single-criterion aggregation structure (like 'utility' function) overrules individual decision criteria and renders their weights of importance as either meaningless or as simple normalizing multipliers. The larger the criterion weight the more valued is criterion performance contribution to the overall aggregate $U$.

But why should the notion of criterion importance be related to or derived from the performance of aggregate superfunction is difficult to answer, decidedly unbeautiful and disharmonious. Maximization of a single-dimensional 'utility' promotes disharmony and disbalance among its components.

Consider the following example. Let there be equal weights $\left(w_{i}=0.2, i=1, \ldots, 5\right)$ of importance of five different decision criteria. In choosing among automobiles we may, for example, consider comfort, quality, price, reliability and mileage to be equally important criteria. Comparing two available automobiles, $\mathrm{A}$ and $\mathrm{B}$, the following five criteria performances are recorded for each:

$$
\begin{array}{ll}
\text { A: }(17 ; 1 ; 1 ; 1 ; 1) & U(\mathrm{~A})=4.2 \\
\text { B: }(4 ; 4 ; 4 ; 4 ; 4) & U(\mathrm{~B})=4.0
\end{array}
$$

The weighted aggregates $U$ achieve values 4.2 and 4.0 for A and B, respectively. A utility maximizer of would choose automobile A with $U=$ 4.2 , other things being equal. He would thus end up with the extravagantly comfortable car of inferior quality, reliability and mileage at a very high price. The whole point of specifying equal importance of all five criteria has been missed. The actual result makes one criterion (comfort) overwhelmingly important and all others unimportant. There is no sense of balance, harmony and equilibrium, maximization of $U$ overrides all such considerations.

Obviously, most rational decision makers search for a specific (here equally weighted) mix of actual criteria performances, ideal if possible, closest to the ideal if necessary. Search for close-to-ideal 
equilibrium is primary concern. The fact that some $U$ is actually maximized at such equilibrium choice is secondary and ex post, a result of conventional tautology: That which maximizes $U$ is preferred and that which is preferred maximizes $U$.

Cultures and companies based on central planning, socialistic command and collective decision making have a large propensity to aggregate, reduce to a single formula, rely on 'overall' utility function, subsume separate criteria within one dominant single measure. Collectives and totalities disregard the individual and his criteria for the sake of some higher, aggregate and collective purpose: the utility function. Freely competing cultures and enterprises stress the individual and his criteria as being autonomous, equal and separately achievable.

\section{Quality}

It is to be expected that other notions based on proximity to ideal, prominence or perfection of achievements and harmony or organization, like notions of quality, truth, goodness, liberty, equality and justice will be strongly related to beauty.

Intuitively and experientially we find products and processes of high quality to be also beautiful, elegant and pleasing, i.e., harmonious, well balanced and complementary in appearance, function and use.

Many of the things we have said about beauty can be repeated for quality. Quality also pertains to objects within a given class and it is related to the class ideal or assemblage of representative standards and perfections. Quality is also definitionally multidimensional and multifaceted. Perception of quality derives from the proximity, approximativeness or resemblance of such ideals. The ranges of quality are related to the remoteness or distance from the ideal. The degrees of quality, as the degrees of beauty, can be assessed, evaluated and measured.

Traditionally however, for example according to J.M. Juran, quality is defined as 'fitness for use' and 'conformance to standards'. Japanese define quality as the totality of characteristics used to determine if and how an intended application has been fulfilled. Another view sees quality as the minimum level of service to satisfy target clientele. The closest and most useful concept of quality is implied by the Japanese term shitsu, implying a balance among measured values. None of these definitions is complete or suitable for our purposes.

What is the difference between quality and the concept of beauty discussed above? It seems that beauty can be perceived for its own sake, i.e., without any reference to its production, use or application. Quality is much more explicitly produced by man and fully intended for the purposeful uses of man. Beauty does not have to be confirmed by use or action, it can be simply contemplated. Quality can only be revealed through use or application towards goals.

A rose or sunset can be beautiful, but we do not necessarily speak of their quality. A specifically bred rose, produced for the purpose of competition or sale to customers, can be a quality rose. Many quality products and processes can be (and often are) beautiful: the two concepts, beauty and quality, emerge through very similar mechanisms. Even the products and processes of nature could be designated as being of quality - if they are applied towards the uses of man.

Beauty is assessed by judgment, quality can only be ascertained by use. Beauty can be assessed as a pure proximity to ideal, quality, because of the subsequent use, has to take the costs into account. Beauty emerges from the quest for perfection, quality emerges from the quest for usable (or affordable) perfection. Beauty is assessed by the observer (or contemplator), quality is brought forth by the user (actor, customer, consumer).

Quality is related more to beauty than to productivity. Both quality and beauty are directly related to human creatively and both are, therefore, readily acceptable. The notion of productivity is of much later origin and is not readily or intuitively accepted by man.

Quality refers to a differentially weighted complex of multiple criteria or dimensions, approximating the ideal complex as closely as possible under the constaints of affordability of use and costs.

Both the ideal and the two constraints of affordability are being continually displaced by the very acts of production and use. The notions of quality, much more intensely so than the relatively stable 
notions of beauty, are therefore rapidly and continually shifting. Pursuits of both beauty and quality are never ending, but the pursuit of quality is aiming at continually changing ideals.

\section{Harmony}

There is a class of naturally or spontaneously produced systems which are not necessarily beautiful or of high quality, but their preservation is even more crucial: the physical, biological and social ecosystems.

Beauty relates to the harmony perceived in things and products. Quality relates to the harmony perceived in the affordable use of things and products. What about the perceived harmony among the processes 'producing' these things and products? These are ecosystems: the primary 'quality' of ecosystems is harmony.

Harmony, as opposed to chaos, conflict or contention, is sustaining, productive and ordering principle. All living systems are dependent and sustained by 'consuming order'. All order is produced by the harmonious concatenation of the production processes - ecosystems. All living systems are dependent on harmony.

Harmony among the production processes is the only source of harmony within the use of products (quality, economy) and the only source of harmony within the products themselves (beauty, culture). Destroying the harmony of the processes, i.e., ecosystems, achieves the 'Los Angeles Effect': it definitionally annihilates the harmony of the products, i.e., quality, economy and beauty.

Harmonious 'social' organization is a network of interactions, reactions and processes involving:

(1) production (poiesis): the rules and regulations guiding the entry of new living components (such as birth, membership, acceptance);

(2) bonding (linkage): the rules guiding associations, functions and positions of individuals during their tenure within organization;

(3) degradation (disintegration): processes associated with the termination of membership (death, separation, expulsion).

All these circularly concatenated processes represent 'productions' of components necessary for other processes, not only the one designated Production. To emphasize this crucial point we speak of poiesis instead of production and autopoiesis instead of self-production. Although in reality hundreds of processes can be so interconnected, the above three-process model represents the minimum conditions necessary for autopoiesis to emerge.

The preservation, sustainment and enhancement of harmony, quality and beauty, if necessary in that very order, is the new challenge and charge of mankind.

The self-producing and self-organizing circle or ecology, economy and ethics must be restored and never broken again.

\section{References}

[1] L. Fusco Girard (1989): The Complex Social Value of Architectural Heritage, ICOMOS Information, no. 1, 1986. Also: Qualità e quantità nello sviluppo urbano. I riflessi sui problemi della valutazione. In P. Nijkamp, H. Voogd \& L. Fusco Girard, Conservazione e Sviluppo, (Franco Angeli, Milano), pp. 11-62.

[2] M. Maruyama (1992): Entropy and Beauty, Human Systems Management, 11 (1992), p. 165.

Milan ZELENY

Graduate School of Business Fordham University at Lincoln Center New York, NY 10023 